Revista Baiana de Saúde Pública
ARTIGO ORIGINAL

\section{EVOLUÇÃO DAS TRANSFERÊNCIAS FINANCEIRAS NO PROCESSO DE DESCENTRALIZAÇÃO DA VIGILÂNCIA SANITÁRIA NO SUS}

Jorge José Santos Pereira Solla Ediná Alves Costa ${ }^{b}$

\begin{abstract}
Resumo
A descentralização do Sistema Único de Saúde vem sendo construída de forma gradativa, regulada pelas Normas Operacionais editadas pelo Ministério da Saúde desde 1991. No entanto, o processo de descentralização da vigilância sanitária - ação específica do setor saúde e competência exclusiva do Estado - não tem acompanhado o mesmo vigor do processo de descentralização da atenção à saúde. Houve limitada indução financeira para municipalização da vigilância sanitária após a Norma Operacional 01/96. As outras normas, mesmo as relacionadas com a atenção básica, não conseguiram articular-se efetivamente com a vigilância sanitária. Este artigo apresenta a evolução das transferências de recursos financeiros no processo de descentralização, no período entre 1998 e 2005 e discute especificamente o financiamento da vigilância sanitária.
\end{abstract}

Palavras-chave: Descentralização. Vigilância Sanitária. Sistema de Saúde.

\section{Abstract}

The decentralization of the Unified Health System has being gradually constructed, regulated through Operational Norms edited by the Brazilian Ministry of Health since 1991. However, the process of decentralization of the Sanitary Surveillance - specific action of the health sector and exclusive capacity of the State - has not followed the same strength of the process of decentralization of the health care. There was limited financial induction for decentralization of the sanitary monitoring for the municipal districts after the Operacional Norm of 1996. The other norms, even the related ones with primary health care, had not articulated themselves effectively with Sanitary Surveillance. This article presents the evolution of the transferences of financial

a Instituto de Saúde Coletiva - Universidade Federal da Bahia (UFBa).

Endereço para correspondência: Rua Marechal Floriano, 41, Apt. 101 - Canela-Salvador-Bahia. CEP: 40.110-010. E-mail: solla.jorge@gmail.co

b Instituto de Saúde Coletiva - Universidade Federal da Bahia (UFBa). 
means in the process of decentralization, in the period between 1998 and 2005 and specifically argues the evolution and trends of the financing of the Sanitary Surveillance.

Key word: Decentralization. Sanitary Surveillance. Health System.

\section{INTRODUÇÃO}

A descentralização no Sistema Único de Saúde (SUS) tem transferido responsabilidades, prerrogativas e recursos para os governos municipais, criando possibilidades de maior autonomia do nível local no uso dos recursos e na definição e implementação de políticas, maior acesso e controle pela população e impacto positivo na gestão e na atenção. ${ }^{1}$

O Brasil é um país continental e complexo, marcado por profundas desigualdades econômicas, sociais, demográficas, culturais e sanitárias. Tal quadro ressalta a importância da descentralização das políticas públicas, inclusive na área de saúde. Além disso, trata-se de um sistema federativo muito particular, no qual os municípios são entes federados, dotados formalmente de autonomia política, administrativa e financeira e protagonistas da gestão da saúde. ${ }^{2}$ São 5.563 municípios, além do Distrito Federal e de Fernando de Noronha, totalizando 5.565 entes federativos locais. A maioria absoluta $(71,3 \%)$ tem menos de 20.000 habitantes e apenas 255 municípios, aqueles com mais de 100.000 habitantes, concentram 53,43\% da população brasileira.

A Constituição ${ }^{3}$ definiu, no Capítulo I, Artigo 198, entre os princípios da gestão do SUS, a descentralização, com direção única em cada esfera de governo e também atribuiu ao município, entre outras competências, prestar serviços de atendimento à saúde da população, com a cooperação técnica e financeira da União e do Estado. De acordo com o mandamento constitucional, a Lei Orgânica da Saúde definiu a descentralização político-administrativa, com direção única em cada esfera de governo, com ênfase na descentralização dos serviços para os municípios.

Ao redesenhar o papel de estados e municípios perante a União, a Constituição também redefiniu o pacto federativo, que, especialmente no setor saúde, "[...] passa a ter como eixo a descentralização das definições políticas, recursos financeiros e fundamentalmente serviços nas instâncias descentralizadas em Estados e municípios". 4:418

Na Constituição, o conceito de saúde foi assumido de forma ampliada, enquanto qualidade de vida. A saúde passou a ser defendida como direito social universal, um direito de cidadania, e as ações e serviços de saúde foram caracterizados como de relevância pública. O direito à saúde deve ser assegurado pelo Estado mediante políticas públicas e açoes consoantes com os objetivos e metas fixados na Constituição. ${ }^{5} \mathrm{~A}$ saúde se insere num conjunto de direitos 
Revista Baiana de Saúde Pública

sociais, cuja garantia necessita da atuação do Estado; a realização desses direitos, ou seja, a passagem da declaração jurídica a sua proteção efetiva requer a ampliação dos poderes do Estado. ${ }^{6}$

A descentralização no setor saúde apresenta potencialmente diversas vantagens nos âmbitos administrativo, político e econômico, entre elas: possibilidade de organizar de forma mais racional o sistema de saúde com base em áreas administrativas locais, particularmente para atenção básica à saúde; contribui para facilitar a coordenação intersetorial; permite soluções locais para problemas relacionados a grandes distâncias, comunicação inadequada e áreas de difícil acesso; pode permitir maiores oportunidades para inovações e aumentar o universo de experiências positivas de gestão; promove um contato mais próximo entre governo e população, melhores condições para formulação de políticas, planos e programas mais realistas e adequados à realidade local; permite melhores condições para incrementar a provisão de atenção à saúde em regiões com baixa cobertura; aquisição local de determinados tipos de insumos estimula a economia na região; facilita a participação da comunidade, permitindo melhorar o controle sobre os recursos aplicados, a aceitabilidade, cooperação e sustentabilidade da política de saúde; possibilita às representações de vários grupos sociais, étnicos, religiosos e políticos em diferentes regiões do país participar mais diretamente das tomadas de decisão sobre as políticas de saúde; e permite que as políticas nacionais penetrem em áreas distantes, onde o suporte para programas nacionais é geralmente mais frágil. $^{7}$

A descentralização do Sistema Único de Saúde (SUS) não é automática; vem sendo construída de forma gradativa, processual, com a progressiva ampliação do número de municípios a assumirem a função de dirigentes plenos das ações e serviços de saúde executados em seu território. $^{5}$

Desde a nova Constituição da República, diversas iniciativas institucionais e legislativas objetivaram criar as condições de viabilização plena do direito à saúde; destacam-se, no âmbito jurídico-institucional, a chamada Lei Orgânica da Saúde (Lei 8.080/90 e a Lei 8.142/ 90) e as Normas Operacionais Básicas (NOB). A Lei Orgânica regulamentou o Sistema Único de Saúde, agregando todos os serviços públicos — federal, estadual e municipal — e os serviços privados contratados/conveniados. As Normas Operacionais Básicas têm sido instrumentos regulamentadores que, progressivamente, têm aprofundado o processo de descentralização da gestão do sistema de saúde e definido as relações entre as três esferas de governo. A NOB 01/91 ${ }^{10}$, ainda editada pela Presidência do Instituto Nacional de Assistência Médica da Previdência Social (INAMPS), estabeleceu a transferência de recursos para estados e municípios por meio de convênios, considerando estes entes federativos como prestadores de serviços. A NOB/SUS 01/93 ${ }^{11}$ criou três alternativas de gestão, expressando graus crescentes de descentralização: incipiente e 
parcial, em que continuavam apenas como prestadores de serviços ao SUS, e a gestão semi-plena, quando pela primeira vez alguns municípios passaram efetivamente a assumir a gestão do sistema de saúde. Esta NOB estabeleceu o funcionamento de instâncias colegiadas de gestão do SUS, as Comissões Intergestores Tripartite (nacional) e Bipartites (estaduais).

Este artigo aborda a descentralização das ações e serviços de saúde a partir das Normas Operacionais Básicas e tem o objetivo de apresentar e discutir a evolução das transferências de recursos financeiros no processo de descentralização e construção do Sistema Único de Saúde, no período de 1998 a 2005, identificando-se especificamente a evolução da descentralização e tendências do financiamento da vigilância sanitária. Os dados foram obtidos do Sistema de Informação Ambulatorial do Sistema Único de Saúde (SIA/SUS) na homepage do DATASUS / Ministério da Saúde (www.datasus.gov.br) e tabulados empregando as ferramentas TABNET e TABWIN, com acessos realizados em março de 2006.

\section{DESCENTRALIZAÇÃO DAS AÇÕES E SERVIÇOS DE SAÚDE NO SUS}

O processo de municipalização das ações e serviços de saúde teve um marco importante em 1994, quando os primeiros municípios assumiram a denominada "gestão semiplena" nos parâmetros da NOB-93. Em 1994, o Decreto n ${ }^{0} 1.232^{12}$, de 30 de agosto de 1994, autorizou o repasse financeiro direto do Fundo Nacional de Saúde para os Fundos Municipais de Saúde, viabilizando o mecanismo necessário para operacionalizar a gestão semi-plena, iniciada em 24 municípios que haviam assumido este compromisso; progressivamente, foi alcançada mais de uma centena de municípios que vieram a demonstrar a viabilidade da construção do SUS. ${ }^{13}$

Ao final de 1996, dois anos depois, já existiam 137 municípios em gestão semiplena de um total de 3.078 habilitados pela NOB-93. Com a NOB- $96^{14}$ houve um grande incremento da descentralização, de forma que em junho de 2000, dois anos após a implantação desta norma operacional (só efetivada em 1998), já eram 5.426 municípios habilitados, dos quais 520 em gestão plena do sistema municipal de saúde (GPSM).

Em setembro de 2004, apenas 15 municípios ainda não tinham sido habilitados, pelo menos em Gestão Plena da Atenção Básica, e mediante decisão da Comissão Intergestores Tripartite (CIT) todos os municípios passaram a ter a responsabilidade definida da gestão da Atenção Básica. Desde outubro de 2004 todos os estados se encontram em gestão plena, mas em janeiro de 2003 eram apenas 13. Em outubro/05 cabia a todos os municípios pelo menos a gestão da atenção básica; 657 municípios estavam em gestão plena do sistema municipal de saúde (GPSM): 429 pela NOB 01/96 14 e 228 conforme a Norma Operacional da Assistência à Saúde - a NOAS $01 / 02^{15}$. 
Revista Baiana de Saúde Pública
Desde o ano de 2003 tem sido observado um grande aumento dos repasses federais para estados e municípios para financiamento do SUS (mais de 32\% até julho de 2005). Isto representa um incremento superior a 5,3 bilhões de reais em 2 anos e meio, saindo de $\mathrm{R} \$ 1$ 1.361.679.173,67 mensais em dezembro de 2002 para $\mathrm{R} \$ 1$ 1.802.915.868,07 em julho de 2005, com R\$1.063.452.196,22 para média e alta complexidade ambulatorial e hospitalar, R\$ 550.540.455,41 para atenção básica e R\$ 188.923.216,44 para o Fundo de Ações Estratégicas (FAEC). Esta indução financeira viabilizou, entre outros resultados, a entrada de todos os estados em gestão plena. A partir de agosto/03, com a entrada de São Paulo, Estado e Capital, em gestão plena, houve grande incremento das transferências diretas para fundos estaduais e municipais. Em outubro/04, com a entrada dos últimos estados em gestão plena, cessou o pagamento direto pelo Ministério da Saúde a prestadores de serviços de saúde, transferindo-se todos os recursos federais para fundos estaduais e municipais (Gráfico 1).

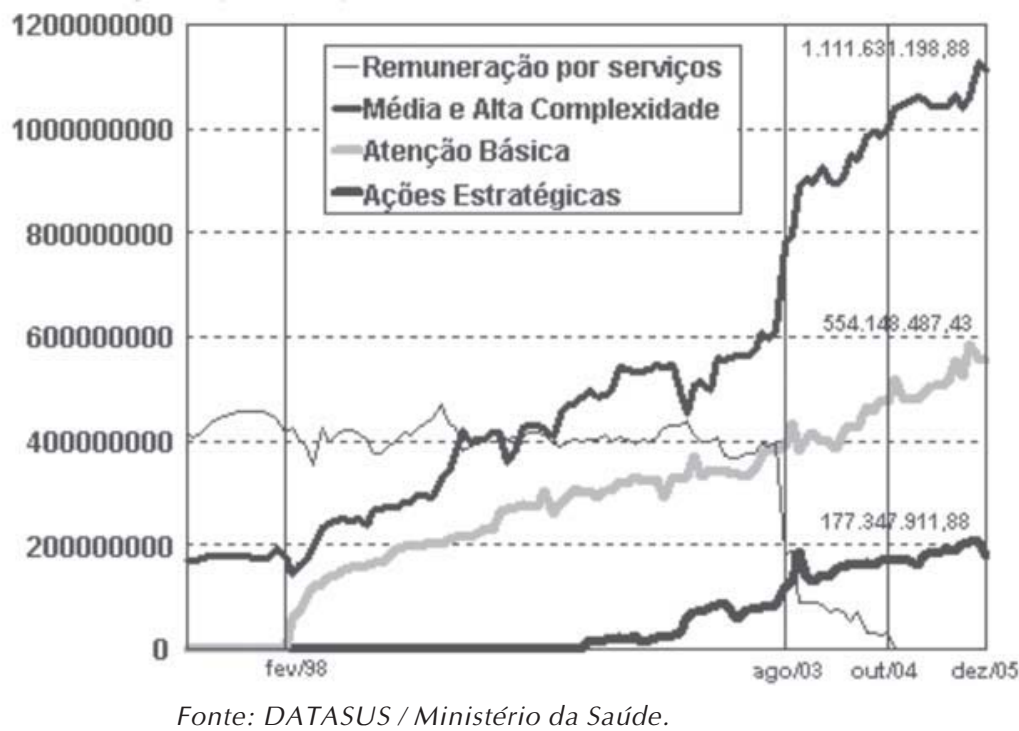

Gráfico 1. Recursos Federais do SUS de acordo com grupo de despesa, Brasil, dezembro/ 1996 a dezembro/2005

Este aumento foi efetivado de forma especial na atenção básica. Comparando o total de recursos federais para atenção básica com os repassados para atenção especializada ambulatorial e hospitalar, isto é, média e alta complexidade (MAC) — sem incluir o Fundo de Ações Estratégicas (FAEC) - evoluiu de 36\% em 2002 para 47\% em 2005. Os recursos para atenção 
básica representavam 19\% do total do MAC em 1998 - ano de implantação do PAB aumentando para 27\% em 1999, ficando entre 33\% e 36\% no período de 2000 a 2002. Em 2003 cresceu para 39\%, em 2004 para 44\% e em 2005 foi igual a 47\%.

Incluindo os recursos do FAEC nos da MAC, o percentual dos recursos para atenção básica em relação a este total ficou entre 33\% e 34\% de 2000 a 2002, aumentando em 2003 para 35\%, em 2004 para 38\%, alcançando 40\% em 2005.

Cabe destacar que, de acordo com dados do Sistema de Informações sobre Orçamentos Públicos em Saúde (SIOPS), apresentados pelo representante do Conselho Nacional de Secretários de Saúde (CONASS) no $2^{\circ}$ Encontro Nacional do Ministério Público em Defesa da Saúde, realizado em setembro de 2005, a evolução dos gastos públicos federais com a saúde, no Brasil, caiu entre 1997 e 2002 de US\$ 89 para US\$ 48 dólares per capita. A partir de 2003, houve uma mudança importante, passando a aumentar anualmente para US\$ 50 neste ano, US\$ 62 em 2004 e com previsão orçamentária de aplicação de US\$ 63 em 2005. ${ }^{16}$

\section{EVOLUÇÃO DA DESCENTRALIZAÇÃO E FINANCIAMENTO DA VIGILÂNCIA SANITÁRIA}

A vigilância sanitária, enquanto ação específica do setor saúde, é explicitada no texto constitucional de 1988 e regulamentada pela Lei Orgânica da Saúde, na qual é estabelecida como "[...] um conjunto de ações capaz de eliminar, diminuir ou prevenir riscos à saúde e de intervir nos problemas sanitários decorrentes do meio ambiente, da produção e circulação de bens e da prestação de serviços de interesse da saúde" ${ }^{\prime \prime 2}$

A descentralização das ações de vigilância sanitária guarda algumas peculiaridades em relação ao processo geral de descentralização do SUS, especialmente no que diz respeito ao "[...] fato de ser atribuição de governo, ou seja, de ser atividade restrita ao setor público"17:299 e de requerer a manutenção de determinadas competências em um órgão central, tais como o registro de produtos e parte das ações em áreas de fronteira, portos e aeroportos, devido à natureza de seus objetos de cuidado que circulam em todo o território nacional e internacional. ${ }^{18}$ As ações de vigilância sanitária, por sua natureza regulatória dirigida à proteção da saúde, constituem tanto uma ação de saúde quanto um instrumento da organização econômica da sociedade. A função protetora abarca não apenas cidadãos e consumidores, mas também os produtores, que atuarão em ambiente de credibilidade, menos exposto à concorrência desleal e à fraude. ${ }^{19}$

Os rumos do processo de descentralização no SUS vêm sendo regulados pelas Normas Operacionais editadas pelo Ministério da Saúde a partir de 1991, e a partir da NOB publicada em 1993 refletem o estágio do processo de negociação entre os gestores das três esferas de governo pactuadas na Comissão Intergestores Tripartite (CIT). 
Revista Baiana de Saúde Pública

As primeiras normas operacionais - mesmo a NOB/93, que teve impacto fundamental ao criar os mecanismos para repasses do fundo nacional de saúde aos fundos estaduais e municipais e efetivar a habilitação de municípios na denominada gestão semi-plena não contribuíram para fazer avançar a descentralização das ações de vigilância sanitária que ficou ao sabor das iniciativas de estados e municípios.

A NOB/96 conferiu maiores responsabilidades ao âmbito municipal, pautou a necessidade de reorientar o modelo de atenção à saúde, impulsionou amplamente o processo de descentralização com duas formas diferenciadas - Gestão Plena da Atenção Básica (GPAB) e Gestão Plena do Sistema Municipal de Saúde (GPSM) — , criou o Piso de Atenção Básica (PAB), ampliando o financiamento para as ações básicas de saúde, estabeleceu o Piso Básico de Vigilância Sanitária (PBVS) e definiu as atribuições de cada esfera de governo no que diz respeito à vigilância sanitária. ${ }^{14,17}$

Contudo, ao não diferenciar o conjunto de atividades e procedimentos de Vigilância Sanitária correspondentes às ações de baixa, média e de alta complexidade, a NOB SUS 01/96 não viabiliza a operacionalização imediata das responsabilidades específicas detalhadas para cada tipo de gestão [...] Na prática encontra-se [sic] VISAs municipais em GPSM sem condições mínimas para o desempenho de suas ações, e, em menor número, VISAs em GPAB plenamente estruturadas para desenvolverem adequadamente suas ações. Mas, certamente, espera-se que as Secretarias Municipais de Saúde em GPSM, avaliadas como possuidoras de efetivo Sistema Local de Saúde, tenham melhores condições para estruturar seus órgãos de Vigilância Sanitária. 17:4;5

É importante destacar que a NOB 01/96 estabeleceu entre as responsabilidades dos municípios em GPSM a prestação de serviços por meio de ações básicas, de média e alta complexidade em vigilância sanitária e, entre as prerrogativas destes municípios, a transferência de recursos para remuneração de serviços de vigilância sanitária de média e alta complexidade e para execução do Programa Desconcentrado de Ações de Vigilância Sanitária, que consiste na prestação de serviços que seriam originalmente de competência do gestor federal. ${ }^{14,20}$ Infelizmente, estes repasses financeiros não foram operacionalizados efetivamente para os municípios em GPSM nos marcos desta norma operacional.

Na NOAS SUS 01/02, a vigilância sanitária aparece apenas entre os requisitos para habilitação dos municípios às condições de gestão estabelecidas, os quais passam a ter que comprovar as condições para desenvolver as ações de VISA definidas. ${ }^{15,17,21}$ 
Tanto no âmbito da NOB SUS 01/96 quanto da NOAS SUS 01/02,

[...] cabe ao Estado a execução de ações de VISA de baixa, média e/ou alta complexidade, apenas quando o município não está habilitado a executá-las, de acordo com as condições de gestão previstas [...] $\mathrm{Na}$ prática, executar ações de vigilância sanitária de alta complexidade requer capacidade técnica e recursos humanos especializados, exigência que muitos municípios de médio e pequeno porte, não têm condições de manter, pelo menos por enquanto, ficando o Estado como o principal executor dessas ações. ${ }^{17: 5}$

Com a estruturação da Agência Nacional de Vigilância Sanitária (ANVISA), a área experimentou significativas mudanças, devido às novas bases de atuação político-institucional, com destaque para os mecanismos de financiamento das ações de vigilância sanitária, mediante o repasse de recursos financeiros da esfera federal para os Estados. Compromissos firmados no Contrato de Gestão — novo instrumento incorporado ao modelo de agência — passaram a exigir, como requisito para seu cumprimento, atuação compartilhada com os serviços estaduais, estabelecendo-se a estratégia de pactuação — mediante o chamado Termo de Ajuste e Metas - e a repartição dos valores arrecadados com as taxas de fiscalização entre o órgão federal e os estados. ${ }^{19}$

No entanto, este esforço da ANVISA para fortalecer o processo de descentralização ficou quase que restrito à cooperação com as Secretarias Estaduais de Saúde. Apenas em 2004 foi efetivamente iniciado o repasse de recursos do governo federal para municípios em gestão plena para financiamento de ações de média e alta complexidade em vigilância sanitária e de valores de taxas de fiscalização recolhidas para a ANVISA.

Nos marcos atuais do processo de descentralização das ações e serviços de saúde espera-se que todos os municípios estruturem minimamente seus serviços de vigilância sanitária e desenvolvam ações de baixa complexidade ou ações básicas de vigilância sanitária. Contudo, apesar da existência de um conjunto importante de municípios que experimentaram grandes avanços na gestão do SUS e na conformação de um sistema municipal de saúde que envolve, inclusive, a Vigilância Sanitária,alguns estudos ${ }^{22-26}$ têm evidenciado a baixa oferta de ações e a precariedade da gestão municipal em vigilância sanitária.

Investigação desenvolvida no Estado do Rio de Janeiro ${ }^{16}$ mostrou que a Secretaria Estadual de Saúde é ainda o executor preferencial das ações de VISA neste estado; não foi encontrada correspondência entre estar em GPSM e uma melhor estruturação da VISA e a não priorização da vigilância sanitária no contexto da descentralização do SUS no Estado, evidenciada 
Revista Baiana de Saúde Pública

pela falta de estruturação dos órgãos locais de VISA. Em outros estados verifica-se a entrega da responsabilidade sobre as ações básicas de VISA para todos os municípios, com desresponsabilização da gestão estadual sobre tal âmbito de intervenção do SUS.

Cabe destacar, no que diz respeito ao financiamento, que até o momento os estados não repassam recursos para os municípios com o objetivo de apoiar as ações em VISA, que conta apenas com recursos federais e municipais para sua realização. Os recursos federais têm estado praticamente estacionários desde a implantação do Piso Básico de Vigilância Sanitária, em 1998. Apesar do aumento registrado a partir de 2003 nos repasses federais para estados e municípios com vistas ao financiamento das ações e serviços de saúde, os recursos repassados para custeio de ações básicas de vigilância sanitária não apresentaram tal evolução, variando de $\mathrm{R} \$$ 43.045.655,61 em 2002 para $R \$ 44.095 .283,01$ em 2005 (Tabela 1).

Tabela 1. Recursos federais repassados para ações básicas de Vigilância Sanitária e proporção em relação ao total de repasses para atenção básica, Brasil, 1998 a 2005

\begin{tabular}{ccc}
\hline Ano & $\begin{array}{c}\text { Recursos para ações } \\
\text { básicas de Vigilância } \\
\text { Sanitária (R\$) }\end{array}$ & $\begin{array}{c}\text { Proporção em relação ao total } \\
\text { de repasses para atenção } \\
\text { básica (\%) }\end{array}$ \\
\hline 1998 & $24.689 .936,64$ & 1,78 \\
1999 & $37.198 .607,68$ & 1,60 \\
2000 & $38.329 .746,75$ & 1,25 \\
2001 & $41.017 .022,85$ & 1,13 \\
2002 & $43.045 .655,61$ & 1,08 \\
2003 & $42.977 .050,75$ & 0,95 \\
2004 & $43.955 .349,83$ & 0,82 \\
2005 & $44.095 .283,01$ & 0,73 \\
\hline
\end{tabular}

Fonte: DATASUS / Ministério da Saúde.

A proporção dos recursos transferidos para financiar ações em vigilância sanitária básica em relação ao total de repasses para atenção básica apresentou redução proporcional e progressiva entre 1998 e 2005: 1,8\% em 1998, 1,0\% em 2002 e 0,7\% em 2005 (Tabela 1).

O número de unidades de vigilância sanitária registradas no SIA/SUS aumentou progressivamente entre novembro/99, quando eram 944, e janeiro/2002, quando já somavam 2.128, sendo 1.180 em janeiro de 2000 e 1.798 em janeiro de 2001. Em janeiro de 2003 houve redução para 2.048 unidades, voltando a aumentar em julho de 2003 para 2.126. 
Os dados atuais do Cadastro Nacional de Estabelecimentos de Saúde $(\mathrm{CNES})^{27}$ apontam a existência de 2.312 unidades de vigilância à saúde, 5.646 unidades com cadastro de especialidade em vigilância sanitária e um conjunto de 6.958 agentes e 1.165 técnicos de vigilância sanitária em atividade.

Esses dados mostram-se significativamente subestimados quando comparados com os obtidos no Censo Nacional dos Trabalhadores de Vigilância Sanitária, realizado em 2004. O censo abrangeu todos os Estados, o Distrito Federal e 4.814 municípios que informaram ter serviços de vigilância sanitária e revelou a existência de 32.135 trabalhadores, dos quais $60 \%$ (19.221) encontram-se em municípios. Quando se examina o tempo em que esses trabalhadores estão nos serviços de vigilância sanitária, pode-se constatar que $67 \%$ tem até 5 anos. Nos pequenos municípios, de até 20 mil habitantes e que detêm 19,3\% do total de trabalhadores, esse percentual se eleva para $78 \%$, indicando um processo de implantação de serviços de vigilância sanitária a despeito da pequena disponibilidade de recursos financeiros. ${ }^{28}$

O repasse de recursos federais para ações de média e alta complexidade (MAC) em vigilância sanitária foi iniciado em agosto de 2000, exclusivamente para os estados, num total mensal de $R \$ 1.787 .359$, que aumentou no mês subseqüente para $R$ \$2.013.921, em fevereiro/ 2001 para $R \$ 2.246 .962$ e em outubro/2001 para $R \$ 2.312 .454$. Em agosto de 2004, já incluindo repasses também para municípios, este montante alcançou o valor mensal de $R \$ 2.341 .439$ e entre fevereiro e dezembro de 2005 ficou mensalmente em $R \$ 2.370 .193,81$, sendo $R \$ 1.667 .626,76$ para os estados e $\mathrm{R} \$ 702.567,05$ para os municípios.

Os recursos referentes a taxas de fiscalização arrecadadas pela ANVISA tiveram repasses esporádicos nos meses de outubro a dezembro nos anos de 2001 a 2003 para os estados. A partir de agosto de 2004 começaram a ser repassados mensalmente para estados e municípios. Entre janeiro e dezembro de 2005 foram repassados mensalmente $R \$ 2.759 .838,90$, sendo $R \$$ 2.144.380,13 para os estados e $\mathrm{R} \$ 615.458,77$ para os municípios.

Em dezembro de 2005, o quadro dos repasses para as capitais, de recursos federais destinados às ações de média e alta complexidade em vigilância sanitária, referentes às taxas de fiscalização arrecadadas pela ANVISA, evidenciava que na região Norte apenas Belém estava incluída e, no Nordeste, ainda faltavam 4 (quatro) capitais - Teresina, Fortaleza, João Pessoa e Salvador; esta última ainda não estava habilitada em GPSM. Por outro lado, Florianópolis, que também não se encontrava em GPSM, vinha recebendo regularmente estes repasses para MAC na área de vigilância sanitária, evidenciando o descompasso existente entre as responsabilidades de gestão na área assistencial e na vigilância sanitária. 
Revista Baiana de Saúde Pública
O número de municípios que registraram procedimentos de vigilância sanitária (VISA) em qualquer quantidade (pelo menos um procedimento), no mínimo em um dos meses do ano aumentou até 2003: 1.609 em 2000, 1.926 em 2001, 2.253 em 2002 e 2.616 em 2003, correspondendo, respectivamente, a 29\%, 35\%, 40\% e 47\% do total de municípios, caindo em 2004 para 2.503 (45\%) e em 2005, para 2.165 (39\% do total) municípios.

O número de procedimentos realizados mostrou tendência semelhante, aumentando até 2003, caindo em 2004 e retomando o aumento em 2005; desta vez apenas no grupo de municípios em GPSM, não sendo observado nos demais, coincidindo com o início dos repasses para financiar ações de média e alta complexidade em VISA em 2004. Em dezembro de 2005 foram registrados 1.810.162 procedimentos de VISA realizados sob gestão municipal; destes $1.315 .579(72,7 \%)$ foram realizados por municípios em GPSM e 494.583 (27,3\%) nos demais municípios (Gráfico 2). A tendência observada na evolução da oferta de ações em VISA no período analisado apresentou comportamento semelhante em todas as regiões do país.

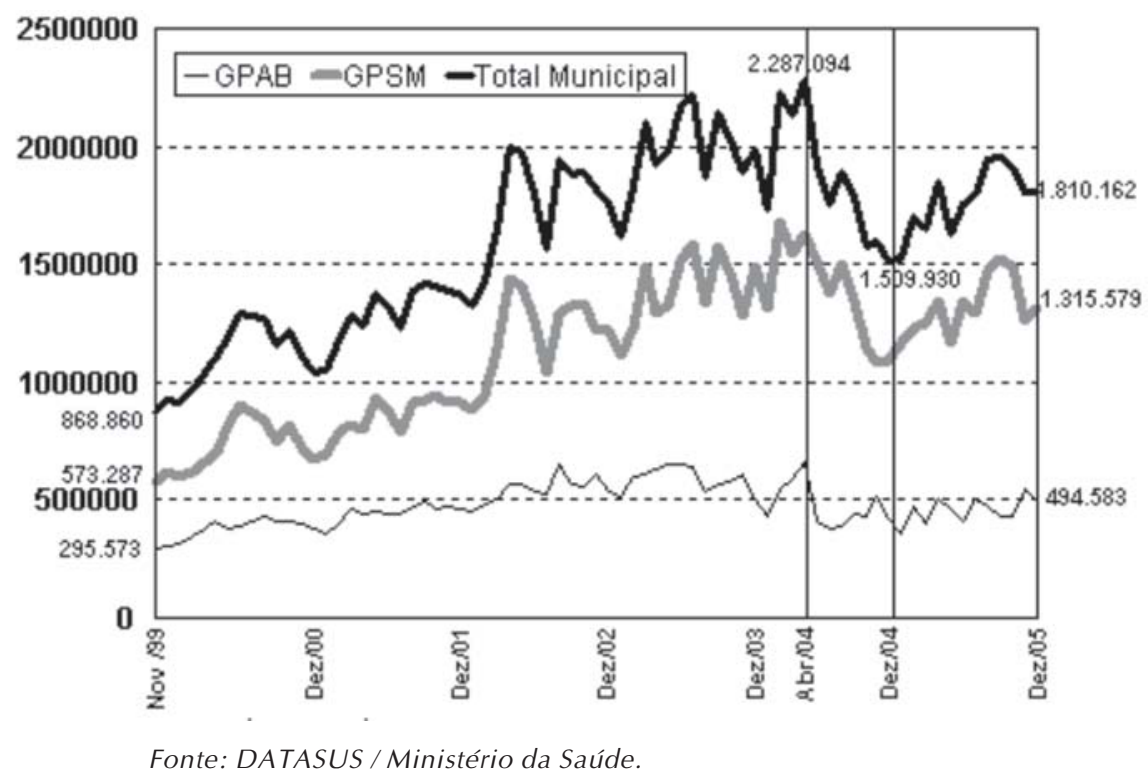

Gráfico 2. Procedimentos básicos em Vigilância Sanitária registrados no SIA/SUS por situação de gestão, Brasil, nov/99 a dez/05

Algumas capitais e grandes municípios ainda registram baixíssima oferta de ações em VISA. Na Tabela 2, apresentam-se as séries históricas de registro de procedimentos de VISA no SUS para as capitais. Em três delas (Belém, Macapá e Cuiabá) não houve registro destes 
procedimentos durante todo o ano de 2005. Chama atenção a ausência de registro de procedimentos de VISA em Goiânia entre 2000 e 2004, a situação do Rio de Janeiro, onde foi registrado apenas um procedimento em 2004 e a significativa redução do registro de ações de VISA em Porto Alegre, a partir de 2004.

Tabela 2. Procedimentos de Vigilância Sanitária realizados em Capitais, Brasil, 2000 a 2005

\begin{tabular}{|c|c|c|c|c|c|c|}
\hline Capital & 2000 & 2001 & 2002 & 2003 & 2004 & 2005 \\
\hline Porto Velho & 111.782 & 166.124 & 117.716 & 86.560 & 22.852 & 33.042 \\
\hline Rio Branco & 6.251 & 6.717 & 5.057 & 7.680 & 6.760 & 10.929 \\
\hline Manaus & 6.982 & 7.557 & 6.688 & 6.437 & 3.317 & 7.008 \\
\hline Boa Vista & 3.075 & 9.527 & 9.295 & 8.700 & 8.420 & 15.462 \\
\hline Belém & 0 & 0 & 1.173 & 3.765 & 0 & 0 \\
\hline Macapá & 2.573 & 31.628 & 18.500 & 7.506 & 19.243 & 0 \\
\hline Palmas & 47.113 & 82.854 & 115.390 & 174.408 & 174.092 & 110.883 \\
\hline São Luís & 16.263 & 16.321 & 17.256 & 16.991 & 34.472 & 50.136 \\
\hline Teresina & 133.879 & 130.038 & 142.669 & 147.837 & 141.792 & 155.582 \\
\hline Fortaleza & 12.688 & 11.741 & 11.729 & 16.127 & 9.592 & 12.403 \\
\hline Natal & 87.790 & 110.072 & 121.483 & 128.069 & 115.580 & 93.704 \\
\hline João Pessoa & 13.146 & 65.898 & 52.298 & 53.297 & 54.519 & 58.715 \\
\hline Recife & 384.087 & 545.392 & 557.647 & 515.763 & 481.478 & 469.846 \\
\hline Maceió & 686.738 & 477.200 & 400.972 & 301.925 & 168.664 & 123.559 \\
\hline Aracaju & 6.589 & 17.953 & 23.294 & 20.357 & 21.248 & 18.028 \\
\hline Salvador & 1.909 & 1.831 & 8.230 & 12.404 & 5.826 & 4.652 \\
\hline Belo Horizonte & 74.195 & 56.848 & 44.193 & 50.689 & 3.511 & 44.811 \\
\hline Vitória & 3.768 & 7.571 & 4.677 & 4.544 & 4.669 & 5.565 \\
\hline Rio de Janeiro & 57.467 & 80.077 & 92.984 & 44.583 & 1 & 6.641 \\
\hline São Paulo & 7.737 & 8.210 & 11.703 & 246.042 & 1.075 .768 & 1.105 .477 \\
\hline Curitiba & 28.584 & 41.090 & 45.486 & 32.008 & 36.190 & 24.520 \\
\hline Florianópolis & 0 & 0 & 1.194 & 1.888 & 5.507 & 6.018 \\
\hline Porto Alegre & 25.951 & 101.482 & 168.169 & 70.697 & 28.973 & 164 \\
\hline Campo Grande & 33.103 & 48.299 & 37.145 & 39.848 & 32.104 & 31.465 \\
\hline Cuiabá & 21.616 & 23.523 & 30.787 & 48.944 & 10.908 & 0 \\
\hline Goiânia & 0 & 0 & 0 & 0 & 0 & 13.771 \\
\hline Brasília & 0 & 0 & 15 & 4 & 7.316 & 2.087 \\
\hline Tot al & 1.773 .286 & 2.047 .953 & 2.045 .750 & 2.047 .073 & 2.472 .802 & 2.404 .468 \\
\hline
\end{tabular}

Fonte: DATASUS / Ministério da Saúde. 
Revista Baiana de Saúde Pública
Na Tabela 3 observa-se o número de procedimentos de VISA registrados anualmente por 1.000 habitantes para as capitais. Os melhores desempenhos registrados em 2005 foram em Palmas (532,66 procedimentos por 1.000 habitantes), Recife $(313,02)$, Teresina $(197,25)$, Maceió $(136,76)$ e Natal $(120,44)$. Com exceção de Salvador e Fortaleza, as capitais nordestinas tiveram um desempenho superior ao das demais regiões.

Tabela 3. Procedimentos de Vigilância Sanitária por 1.000 habitantes realizados emCapitais, Brasil, 2000 a 2005

\begin{tabular}{|c|c|c|c|c|c|c|}
\hline Capital & 2000 & 2001 & 2002 & 2003 & 2004 & 2005 \\
\hline Porto Velho & 334,02 & 485,37 & 338,42 & 244,54 & 63,47 & 88,37 \\
\hline Rio Branco & 24,70 & 25,69 & 18,89 & 27,97 & 24,03 & 35,75 \\
\hline Manaus & 4,97 & 5,20 & 4,49 & 4,21 & 2,12 & 4,26 \\
\hline Boa Vista & 15,33 & 45,69 & 43,33 & 39,36 & 37,01 & 63,85 \\
\hline Belém & 0,00 & 0,00 & 0,89 & 2,81 & 0,00 & 0,00 \\
\hline Macapá & 9,08 & 106,89 & 60,34 & 23,62 & 58,32 & 0,00 \\
\hline Palmas & 343,00 & 549,13 & 716,09 & 1012,96 & 950,39 & 532,66 \\
\hline São Luís & 18,69 & 18,36 & 19,03 & 18,40 & 36,66 & 51,22 \\
\hline Teresina & 187,15 & 178,41 & 192,79 & 196,73 & 185,87 & 197,25 \\
\hline Fortaleza & 5,93 & 5,38 & 5,28 & 7,15 & 4,18 & 5,22 \\
\hline Natal & 123,25 & 152,42 & 165,39 & 171,95 & 153,07 & 120,44 \\
\hline João Pessoa & 21,99 & 108,48 & 84,48 & 84,75 & 85,37 & 88,85 \\
\hline Recife & 269,93 & 379,49 & 384,81 & 352,94 & 326,77 & 313,02 \\
\hline Maceió & 860,83 & 583,77 & 481,21 & 355,32 & 194,73 & 136,76 \\
\hline Aracaju & 14,28 & 38,34 & 49,14 & 42,43 & 43,76 & 36,16 \\
\hline Salvador & 0,78 & 0,74 & 3,27 & 4,85 & 2,25 & 1,74 \\
\hline Belo Horizonte & 33,14 & 25,17 & 19,34 & 21,98 & 1,51 & 18,87 \\
\hline Vitória & 12,89 & 25,58 & 15,62 & 15,01 & 15,26 & 17,76 \\
\hline Rio de Janeiro & 9,81 & 13,58 & 15,66 & 7,46 & 0,00 & 1,09 \\
\hline São Paulo & 0,74 & 0,78 & 1,10 & 23,04 & 100,04 & 101,16 \\
\hline Curitiba & 18,01 & 25,36 & 27,66 & 19,15 & 21,32 & 13,95 \\
\hline Florianópolis & 0,00 & 0,00 & 3,31 & 5,12 & 14,59 & 15,17 \\
\hline Porto Alegre & 19,07 & 73,90 & 121,56 & 50,71 & 20,63 & 0,11 \\
\hline Campo Grande & 49,88 & 71,10 & 53,64 & 56,44 & 44,63 & 41,97 \\
\hline Cuiabá & 44,72 & 47,72 & 61,54 & 96,32 & 21,14 & 0,00 \\
\hline Goiânia & 0,00 & 0,00 & 0,00 & 0,00 & 0,00 & 11,47 \\
\hline Brasília & 0,00 & 0,00 & 0,01 & 0,00 & 3,28 & 0,89 \\
\hline Total & 43,83 & 49,91 & 49,20 & 48,62 & 58,01 & 54,89 \\
\hline
\end{tabular}

Fonte: DATASUS / Ministério da Saúde. 


\section{CONSIDERAÇÕES FINAIS}

Observa-se que a descentralização da assistência, da vigilância sanitária e da vigilância epidemiológica e controle de doenças se deram em tempos e formatos diferentes. Verifica-se limitada indução financeira para municipalização da VISA após a NOB 01/96; a NOAS e movimentos normativos posteriores, mesmo os relacionados com a atenção básica, não conseguiram articular-se efetivamente com a VISA.

Com base nos dados analisados, mesmo considerando a existência de sub-registro das ações de vigilância sanitária no SIA/SUS, e frente à baixa oferta de ações e capacidade de gestão em VISA, inclusive em grandes municípios e capitais, e a importância que parecem ter tido os repasses para financiamento de ações de média e alta complexidade em VISA como indutor para a ampliação das ações nos municípios em GPSM, questiona-se a possibilidade de estar em curso certa tendência à "estagnação" da VISA nos municípios, com comportamento diferenciado naqueles em gestão plena, onde parece indicar um aumento dos procedimentos de VISA registrados em 2005. Ao se refletir sobre as questões do financiamento em contraposição ao dinamismo evidenciado na incorporação de trabalhadores de vigilância sanitária também se questiona o elenco e a qualidade das ações e serviços que poderão permanecer no modelo tradicional, com escassa efetividade, devido aos limites do financiamento.

A gestão em vigilância sanitária é tema recente na agenda da saúde. Em face do debate em torno da necessidade de um novo modelo de atuação na perspectiva da construção de um Sistema Nacional de Vigilância Sanitária integrado no SUS e de acordo com seus princípios doutrinários e organizativos, torna-se imperioso discutir sobre como financiá-lo e como qualificar sua capacidade de gestão de modo a que cumpra sua função de proteger e promover a saúde da população.

\section{REFERÊNCIAS}

1. Hortale VA, Comil E M, Pedroza M. Desafios na construção de um modelo para análise comparada da organização de serviços de saúde. Cadernos de Saúde Pública 1999;15:79-88.

2. Rocha JC. Direito da Saúde: Direito Sanitário na perspectiva dos interesses difusos e coletivos. São Paulo: LTR; 1999.

3. Brasil. Constituição de 1988. $17^{\text {a }}$ ed. Atualizada em 2001. Brasília: Câmara dos Deputados; 2001.

4. Gerschman S. Municipalização e inovação gerencial: um balanço da década de 1990. Ciência \& Saúde Coletiva 2001;6:417-34. 
Revista Baiana de Saúde Pública
5. Weichert MA. Saúde e Federação na Constituição Brasileira. Rio de Janeiro: Lúmen Júris; 2004.

6. Bobbio N. A era dos direitos. Rio de Janeiro: Campus; 1992.

7. Araújo Júnior JL do AC. Decentralization in the Health sector. The Brazilian process, issues and problems, 1988 - 1994 [Dissertação]. Leeds (U.K.): University of Leeds; 1994.

8. Brasil. Lei n. 8.080. Dispõe sobre as condições para a promoção, proteção e recuperação da saúde, a organização e o funcionamento dos serviços correspondentes. Brasília (DF): Diário Oficial da União; 20 set. 1990.

9. Brasil. Lei $n^{\circ} 8.142$, de 19 de dezembro de 1990. Dispõe sobre a participação da comunidade na gestão do Sistema Único de Saúde (SUS) e sobre as transferências intergovernamentais de recursos financeiros na área de saúde. Brasília (DF): Diário Oficial da União; 31 dez.1990.

10. Brasil. Ministério da Saúde. Instituto Nacional de Assistência Médica da Previdência Social (INAMPS). Norma Operacional Básica SUS 1/91. Resolução 273/91. Brasília (DF): Diário Oficial da União; 18 jul. 1991. p. 14.216-9.

11. Brasil. Ministério da Saúde. Descentralização das ações e serviços de saúde. A ousadia de cumprir e fazer cumprir a lei. NOB-SUS 01/93. Brasília (DF); 1993.

12. Brasil. Decreto $n^{0} 1.232$, de 30 de agosto de 1994. Dispõe sobre as condições e a forma de repasse regular e automático de recursos do Fundo Nacional de Saúde para os fundos de saúde estaduais, municipais e do Distrito Federal, e dá outras providências. Brasília (DF): Diário Oficial da União; 31 ago. 1994.

13. Campos CR. A produção da cidadania - construindo o SUS em Belo Horizonte. In: Campos CR, Malta DC, Reis AF, Merhy EE, organizadores. Sistema Único de Saúde em Belo Horizonte: reescrevendo o público. São Paulo: Xamã; 1998. p. 11-30.

14. Brasil. Ministério da Saúde. Norma Operacional Básica do SUS: NOB / SUS - 01/96. Brasília (DF); 1996.

15. Brasil. Ministério da Saúde. Regionalização da assistência à saúde. Aprofundando a descentralização com eqüidade no acesso. Norma Operacional da Assistência à Saúde - NOAS-SUS 01/2002 (Portaria MS/ GM n. 373, de 27 de fevereiro de 2002 e regulamentação complementar). Brasília; 2002. 
16. Lopes CR. Palmas para a luta do MP! Radis 2005;39:11.

17. Cohen MM, Moura ML de O, Tomazelli JG. Descentralização das ações de Vigilância Sanitária nos municípios em Gestão Plena, Estado do Rio de Janeiro. Revista Brasileira de Epidemiologia 2004;7:290-301.

18. Costa EA. Vigilância Sanitária: proteção e defesa da saúde. São Paulo: Sobravime; 2004.

19. Costa EA. Vigilância Sanitária: proteção e defesa da saúde. In: Rouquayrol MZ, Almeida Filho N, organizadores. Epidemiologia \& Saúde. Rio de Janeiro: Medsi; 2003. p. 357-387.

20. Andrade LOM. SUS passo a passo: gestão e financiamento. São Paulo: Hucitec/UVA; 2001.

21. Brasil. Ministério da Saúde. Regionalização da assistência à saúde. Aprofundando a descentralização com eqüidade de acesso. Norma Operacional da Assistência à Saúde - NOAS-SUS 01/2001 (Portaria MS/ GM n. 95, de 26 de janeiro de 2001 e regulamentação complementar). Brasília; 2001.

22. Cunha I de CA, Solla JJSP. Implantação da Vigilância Sanitária em Vitória da Conquista, Bahia. Divulgação em Saúde para Debate $2001 ; 25: 28-36$.

23. Freitas RM, Miranda EP. Vigilância Sanitária: reformulando práticas. In: Campos CR, Malta DC, Reis AF, Merhy EE, organizadores. Sistema Único de Saúde em Belo Horizonte: reescrevendo o público. São Paulo: Xamã; 1998. p. 347-63.

24. Rego AM, Lenzi MM, Bagatin RCS, Zappe R de L. Avaliação Sanitária responsabilidade compartilhada. In: Ducci L, Pedotti MA, Simão MG, Moysés SJ, organizadores. Curitiba: a saúde de braços abertos. Rio de Janeiro: CEBES; 2001. p.145-55.

25. Tierling HSM. Municipalização da Vigilância Sanitária. In: Pimenta AL, organizadora. Saúde e humanização: a experiência de Chapecó. São Paulo: Hucitec; 2000. p.215-22.

26. Juliano IA, Assis MMA. A vigilância sanitária em Feira de Santana no processo de descentralização da saúde (1998-2000). Ciência \& Saúde Coletiva 2004;9:493-505.

27. Brasil. Ministério da Saúde. Secretaria de Atenção à Saúde. Homepage do Banco de Dados do Sistema Único de Saúde - DATASUS. Extraído de [www.datasus.gov.br], acesso em [13 de março de 2006]. 
Revista Baiana de Saúde Pública
28. Brasil. Ministério da Saúde. Homepage da Agência Nacional de Vigilância Sanitária (ANVISA). Extraído de [www.anvisa.gov.br], acesso em [19 de maio de 2006].

Recebido em 09.04.2007 e aprovado em 30.05.2007. 